\title{
Prognostic applicability of simplified acute physiology score (saps 3) in critically ill adult surgical patients in a tertiary Indian hospital: A preliminary study
}

\author{
Dheeraj Kapoor $^{1}$, Meghana Srivastava ${ }^{2}$, Jasveer Singh ${ }^{3, *}$, Komal Aggarwal ${ }^{4}$, Manpreet Singh ${ }^{5}$ \\ 1,3,5 Associate Professor, Dept. of Anaesthesia and Intensive Care, ${ }^{4} \mathrm{PG}$ Trainee, Dept. of General Surgery, Government \\ Medical College and Hospital, Chandigarh, India ${ }^{2}$ Anaesthesia Specialist, Dept. of Anaesthesia, Princess of Wales Hospital, \\ Bridgend, United Kingdom \\ *Corresponding Author: \\ Email: drjassy18@gmail.com
}

Received: $10^{\text {th }}$ December, 2017

Accepted: $05^{\text {th }}$ February, 2018

\begin{abstract}
Introduction and Aims: Prognostic indices are infrequently practiced in surgical intensive care units (SICU) of Indian tertiary care hospitals to assess the overall outcomes. Simplified Acute Physiology Score 3 (SAPS 3) prognostic system is now practiced globally and comprises easily measurable parameters on admission of patient in intensive care unit (ICU). The objective of the present study was to evaluate SAPS 3 index as a predictor of mortality in postoperative critically ill surgical patients admitted to ICU in a northern Indian setup.

Materials and Methods: This prospective observational study was performed in the ICU of a tertiary hospital in northern India. SAPS 3 global model was applied to measure the predicted ICU mortality. Standardized mortality ratio (SMR) was computed by comparing the observed and predicted mortality rates. To predict ICU mortality the discrimination and calibration properties of the SAPS 3 index were analysed. Data were prospectively collected at the time of admission of surgical patients in ICU. Estimated mortality rates were measured by SAPS 3 scores. Discrimination was estimated by area under receiver operating characteristic (AUROC) curves. Calibration was interpreted by Hosmer-Lemeshow goodness-offit C-statistic test to appraise the agreement between observed and expected number of survivors and non- survivors in alliance to the probability of death. In this analysis, P > 0.05 denotes good test adjustment.

Results: A total of 55 postoperative patients were included over 3 months period. The observed ICU mortality was $17.1 \%$. Standardised mortality ratio (SMR) was 1.07 . The SAPS 3 score of 42 showed sensitivity and specificity of $83.3 \%$ and $45.8 \%$ respectively. SAPS 3 global index had fair discrimination with an area under the receiver operating characteristic curve (AUROC) of 0.743 (CI 0.55-0.93). Patient calibration by Hosmer-Lemeshow test displayed good adjustment (P -0.388 and $\left.\mathrm{X}^{2}-6.32\right)$.

Conclusion: This group of postoperative SICU patients, the performance of SAPS3 prediction model showed reasonable discrimination and good calibration in predicting mortality risk in northern Indian tertiary hospital.
\end{abstract}

Keywords: Calibration, Discrimination, Intensive care unit, Mortality, Validation.

\section{Introduction}

In the modern era, the intensive care units (ICUs) constitute a prodigious segment of health care resources owing to the expansive technical framework and the health care professionals involved. Proficient management of these resources is the basis of providing quality of care to the admitted patients. In the critical care setups, the prognostic indices such as Simplified Acute Physiology Score (SAPS) and Acute Physiology and Chronic Health Evaluation (APACHE) are commonly endorsed to quantify the severity of the admitted patients. ${ }^{1,2}$ These indices may indirectly ascertain the performance of the critical care providers, the cost-benefit ratio of the critical units and can provide the guide for further allotment of health care personnel and equipments. ${ }^{3}$ In recent years, the number of surgical patients admitted to intensive care units (ICUs) has heightened dramatically. ${ }^{4}$ The surgical outcome of these patients is immensely influenced by their preoperative physiologic status, the nature of surgical intervention and the postoperative care. ${ }^{5}$ Hence the predictive data of risk for morbidity and mortality for this subset of patients is of utmost importance. ${ }^{6}$ The subset of patients undergoing surgical interventions were initially evaluated and stratified by the grading proposed by the American Society of Anaesthesiologists (ASA), which provides the physical status of the patients prior to the surgery and hence have limited predictability of the clinical outcome. Therefore more established and validated prognostic indicators and predictability outcome measures are required.

The evolution of the SAPS 3 system comprises the evaluation of a colossal data from more than 300 critical care units. ${ }^{7,8}$ It has been observed that SAPS3 has better discriminatory assessment when compared to SAPS II and APACHE II and hence the later systems being discouraged in clinical practice. SAPS 3 surpass other prognostic indices as this model evaluates the data entirely in the first hour after ICU admission and hence ideal for ICU screening and reflects the real clinical status of the patient.SAPS3 index is simple and easy to calculate and should be 
routinely used in surgical patients in ICUs for risk stratification and for predicting the clinical outcomes and hence may act as a functional stratagem for this subset of patient. Though various studies have validated SAPS 3 prognostic system and subsequently integrated in ICU protocols, ${ }^{7-11}$ only few developed it in surgical patients in critical care settings., ${ }^{40,12-}$ ${ }^{14}$ SAPS 3 is extensively used both in Europe and America continent in the ICUs ${ }^{10}$ while evidence is sporadic is India in clinical practice. ${ }^{15,16}$ Therefore, the objective of the present study was to analyse SAPS 3 index as a predictor of mortality in postoperative critically ill patients admitted to ICU in an northern Indian setup.

\section{Materials and Methods}

The present prospective observational study was performed in a intensive care unit of tertiary hospital in northern India with a total of 10 beds, coordinated primarily by a qualified intensivists and critical care nursing staff under department of Anaesthesia and Intensive care.

After approval of the present study by the Institutional Ethics Committee (IEC) (No.GMCHTA-1-2015/04983), a written informed consent was obtained from the legal representative of the patients. The present study did not involve any invasive procedures. Confidentiality of the patient was maintained and patient or his/ her relative were given right to opt out of study at any given point of time.

All consecutive adult surgical patients admitted to ICU in a 3 month period were included in the study. Patients younger than 18 years, stay in ICU less than 24 hours, readmissions and those admitted only for diagnostic interventions were excluded from the study. Patients follow up was done till discharge from the ICU/ hospital or had expired. Data were collected and recorded in the first hour postadmission in ICU by independent investigator. All patients were managed as per ICU protocol of the hospital and no intervention in the therapeutic regimen were made.

The SAPS 3 prognostic index comprises 20 variables, measured on admission in ICU. The parameters are grossly divided into three parts, demographic variables, cause for admission in the ICU, and physiologic parameters. (Appendix 1) A score was assigned to each parameter, and after taking the airthmetic summation of all subscores, the SAPS 3 score is calculated. A total SAPS 3 score of 16 and 217 as the lowest and highest score respectively. Physiologic parameters included were systolic blood pressure, heart rate, temperature, oxygenation, arterial $\mathrm{pH}$, haematocrit, leukocytes, platelets, creatinine, bilirubin, and Glasgow coma scale (GCS).
Demographic variables were showed as mean \pm standard deviation, median (25-75percentile), or percentage and frequency. To analyse the discrimination, defined as ability to classify nonsurvivors and survivors, sensitive and specific tests were applied for different SAPS 3 scores. ROC (Receiver Operating Characteristics) curve was plotted and the area under the curve was calculated. The optimum discriminating value was chosen by the calculated maximum specificity and sensitivity. Cutoff point was taken as the higher value derived from this result. Confidence intervals (CI) of 95\% were calculated for true and false positive rates and for the correct stratification of the outcome. The HosmerLeme show goodness-of-fit C-statistic test was applied to appraise the concordence between the observed and expected number of survivors and nonsurvivors respectively, in association to the likelihood of death. In this analysis, P> 0.05 denotes good test adjustment. The standardized mortality ratio (SMR) was studied by ratio of the observed and predicted mortality rate.

\section{Results}

Total of 55 patients were admitted in the ICU in the study time period. Twenty patients were excluded as they were admitted exclusively with medical problems. Rest of the 35 patients admitted have undergone surgical intervention and were included in the study. Out of these 35 patients, 5 patients were excluded as they were not fulfilling the inclusion criteria [readmission $(n=1),<24$ hour ICU stay $(n=2)$, admitted exclusive for diagnostic intervention $(n=2)]$. Mean patient age was $44.30 \pm 17.48$ years, out of which 20\% were females (Table 1). Patient who underwent gastrointestinal surgeries predominated (76.6\%), followed by orthopaedic surgeries (10\%) (Table 1). In the present study, the lowest and highest SAPS 3 score was 22 and 70 respectively, with a mean of $45.36 \pm 11.97$ (Table 1). The observed and predicted mortality was $17.1 \%$ and $15.84 \%$ respectively. Standardised mortality ratio (SMR) was 1.07. The SAPS 3 score of 42 showed specificity and sensitivity of $45.8 \%$ and $83.3 \%$ respectively, for ICU mortality with an area under the receiver operating characteristic curve (AUROC) of $0.743,95 \%$ CI (0.55-0.93), thus reasonably discriminating the mortality in the study population (Fig. 1). Higher rate of survival was observed in patients whose SAPS 3 score was $\leq 42$. Among patients with SAPS 3 scores $>42,83.3 \%$ were non-survivors versus $54.2 \%$ of survivors (Fig. 2). Patient calibration in accordance with the Hosmer-Lemeshow test revealed good adjustment (P -0.388 and $\mathrm{X}^{2}$-6.32) (Fig. 3). 
Table 1: Patient distribution characteristics, SAPS 3 score, type of surgery and ICU mortality

\begin{tabular}{|l|c|}
\hline \multicolumn{1}{|c|}{ Characteristics } & Parameter \\
\hline Patient numbers & 30 \\
\hline Elective surgery & $56.7 \%$ \\
\hline Age (years) & $44.3 \pm 17.47$ \\
\hline SAPS 3 score & $45.36 \pm 11.97$ \\
\hline Before surgery in hospital days & $3.57(1-5)$ \\
\hline Type of surgery & \\
\hline Gastrointestinal & $76.6 \%$ \\
\hline Orthopaedic & $10 \%$ \\
\hline Others & $13.4 \%$ \\
\hline ICU Mortality & $17.1 \%$ \\
\hline
\end{tabular}

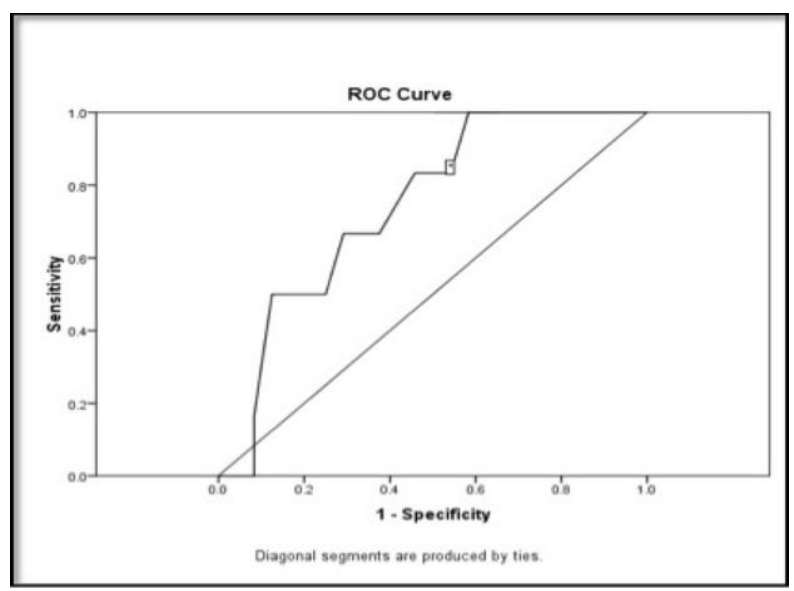

Fig. 1: AUROC Curve for prediction of hospital mortality

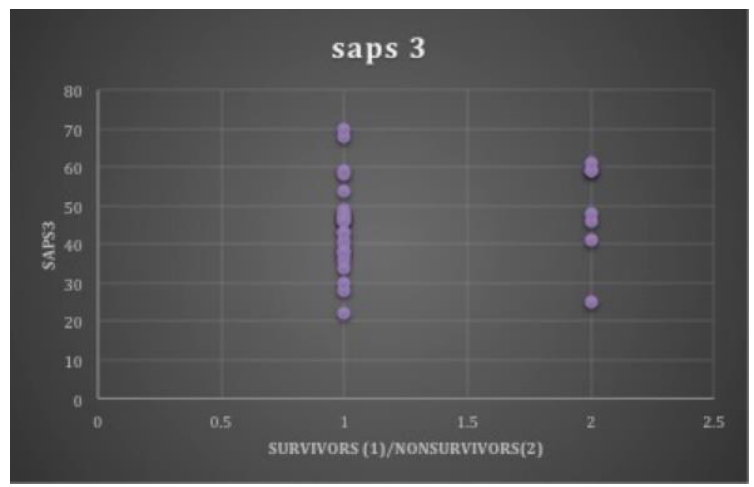

Fig. 2: Patient distribution according to SAPS 3 score (survivors 1 and non-survivors 2)

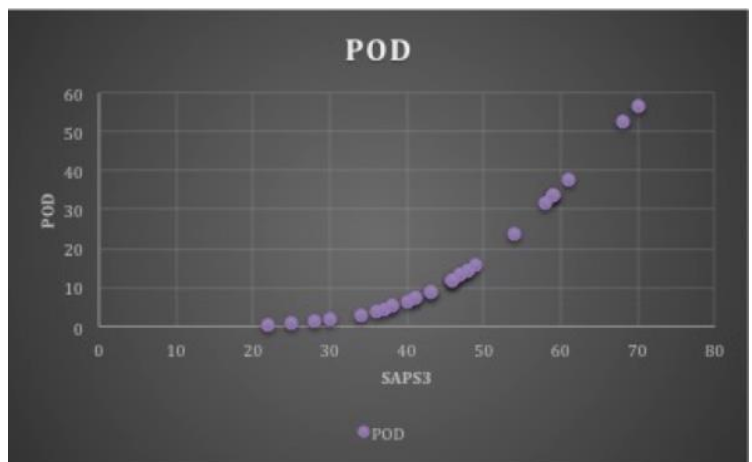

Fig. 3: Relationship between the SAPS 3 score and the probability of death (POD) 
Appendix 1: SAPS 3 prognostic index variables

\begin{tabular}{|c|c|c|c|c|c|}
\hline \multicolumn{2}{|c|}{$\begin{array}{l}\text { Demographics/previous } \\
\text { health status }\end{array}$} & \multicolumn{2}{|l|}{ Diagnostic category } & \multicolumn{2}{|c|}{$\begin{array}{l}\text { Physiologic parameters on } \\
\text { admission }\end{array}$} \\
\hline Parameters & Score & Parameters & Score & Parameters & Score \\
\hline Age & & \begin{tabular}{|l|} 
Urgency \\
\end{tabular} & & Heart rate & \\
\hline$<40$ & 0 & Non-surgical & 5 & $<120$ & 0 \\
\hline$\geq 40<60$ & 5 & Elective & 0 & $\geq 120<160$ & 5 \\
\hline$\geq 60<70$ & 9 & Emergency & 6 & $\geq 160$ & 7 \\
\hline$\geq 70<75$ & 13 & $\begin{array}{l}\text { Scheduled } \\
\text { admission }\end{array}$ & 0 & $\begin{array}{l}\text { Systolic blood } \\
\text { pressure }\end{array}$ & \\
\hline$\geq 75<80$ & 15 & $\begin{array}{l}\text { Non-scheduled } \\
\text { admission }\end{array}$ & 3 & $<40$ & 11 \\
\hline$\geq 80$ & 18 & $\begin{array}{l}\text { Reason for } \\
\text { admission }\end{array}$ & & $\geq 40<70$ & 8 \\
\hline $\begin{array}{l}\text { In-hospital } \\
\text { days before } \\
\text { ICU }\end{array}$ & & Neurologic & & $\geq 70<120$ & 3 \\
\hline$<14$ & 0 & Seizures & 4 & $\geq 120$ & 0 \\
\hline$\geq 14-28$ & 6 & $\begin{array}{l}\text { Coma, confusion, } \\
\text { agitation }\end{array}$ & 4 & $\begin{array}{l}\text { Glasgow Coma Scale } \\
\text { (GCS) }\end{array}$ & \\
\hline$\geq 28$ & 7 & Focal deficit & 7 & $3-4$ & 15 \\
\hline Comorbidities & & $\begin{array}{l}\text { Intracranial mass } \\
\text { effect }\end{array}$ & 11 & 5 & 10 \\
\hline Others & 0 & Infection & & 6 & 7 \\
\hline Chemotherapy & 3 & Nosocomial & 4 & $7-12$ & 2 \\
\hline ICC NYHA IV & 6 & Respiratory & 5 & $\geq 13$ & 0 \\
\hline $\begin{array}{l}\text { Hematologic } \\
\text { neoplasia }\end{array}$ & 6 & Others & 0 & Temperature & \\
\hline Cirrhosis & 8 & Abdomen & & $<34.5$ & 7 \\
\hline AIDS & 8 & Acute abdomen & 3 & $\geq 34.5$ & 0 \\
\hline Metastasis & 11 & Severe pancreatitis & 9 & Oxygenation & \\
\hline $\begin{array}{l}\text { Vasoactive } \\
\text { drugs }\end{array}$ & & Liver failure & 6 & $\begin{array}{l}\text { Mechanical ventilation } \\
\mathrm{PaO} 2 / \mathrm{FiO} 2<100\end{array}$ & 11 \\
\hline Yes & 0 & Others & 0 & $\begin{array}{l}\text { Mechanical ventilation } \\
\mathrm{PaO} 2 / \mathrm{FiO} 2 \geq 100\end{array}$ & 7 \\
\hline No & 3 & Cardiac cause & & $\begin{array}{l}\text { Without mechanical } \\
\text { ventilation } \mathrm{PaO} 2<60\end{array}$ & 5 \\
\hline Origin & & Arrhythmia & 5 & $\begin{array}{l}\text { Without mechanical } \\
\text { ventilation } \mathrm{PaO} 2>60\end{array}$ & 0 \\
\hline $\begin{array}{l}\text { Operating } \\
\text { room }\end{array}$ & 0 & Hemorrhagic shock & 3 & pH & \\
\hline $\begin{array}{l}\text { Emergency } \\
\text { Room (ER) }\end{array}$ & 5 & $\begin{array}{l}\text { Non-hemorrhagic } \\
\text { hypovolemic shock }\end{array}$ & 3 & $\leq 7.25$ & 3 \\
\hline Other ICU & 7 & Distributive shock & 5 & $>7.25$ & 0 \\
\hline \multirow[t]{7}{*}{ Others } & 8 & Type of surgery & & Bilirubin & \\
\hline & & Transplantation & 11 & $<2$ & 0 \\
\hline & & Trauma & 8 & $\geq 2<6$ & 4 \\
\hline & & MR without valve & 6 & $\geq 6$ & 5 \\
\hline & & Stroke surgery & 5 & Creatinine & \\
\hline & & Other & 0 & $<1.2$ & 0 \\
\hline & & ICU admission & 16 & $\geq 1.2-<2.0$ & 2 \\
\hline & & & & $\geq 2.0<3.5$ & 7 \\
\hline & & & & $\geq 3.5$ & 8 \\
\hline & & & & Leukocvtes & \\
\hline & & & & $<15,000$ & 0 \\
\hline
\end{tabular}




\begin{tabular}{|l|l|l|c|}
\hline \multirow{4}{*}{} & & $\geq 15,000$ & 2 \\
\cline { 2 - 3 } & & Platelets & \\
\cline { 2 - 3 } & & $<20,000$ & 13 \\
\cline { 2 - 3 } & & $\geq 20,000<50,000$ & 8 \\
\cline { 2 - 3 } & & $\geq 50,000<100,000$ & 5 \\
\cline { 2 - 3 } & & $\geq 100,000$ & 0 \\
\hline
\end{tabular}

\section{Discussion}

Contemporary intensive care unit (ICU) utilizes a sizeable proportion of health resources owing to the advancement in the technology for diagnostic and therapeutic interventions, required for the critically ill patients. Prognostic indices to determine the severity of the illness are adjudged as a major factor to determine the cost-benefit ratio of these units. These indices may guide for appropriate allocation of specialized equipments and health care personal required in critical care units. American Society of Anaesthesiologists (ASA) physical status has been extensively used till now to evaluate the health status of surgical patients. However, ASA physical status dispense information of only patient health status prior to the surgical intervention, hence poorly correlates with the severity risk of surgical patients admitted in ICU.

For many years, SAPS II and APACHE II prognostic models are frequently used scoring system in ICU. ${ }^{13}$ SAPS 3 is currently the most widely practiced prognostic severity index in ICU setups. ${ }^{10}$ In the SAPS 3 model the whole data is recorded within the first hour of the ICU admission in contrast to the previous versions, where data collection is done within first 24 hour of the admission. ${ }^{7,8}$ In addition, unlike previous versions, in the SAPS 3 model, most of the data of the patient admitted in ICU is recorded from the information provided prior to ICU admission, hence making this model a potential tool for triage of ICU patients. ${ }^{13}$ Moreover, data obtained after first hour of ICU admission of patient may not be labled precisely for screening, as it largely reflects the ongoing ICU care administered to the admitted patients. ${ }^{13}$ Previous severity scores are largely computed on data observed after 24 hours of ICU admission and may be affected by so called "Boyd and Grounds effect". ${ }^{8}$ This effect explains that the predicted mortality of patient admitted in ICU is directly linked to the abnormal physiological values and subsequent severity scores, in the first 24 hours post ICU admission. ${ }^{17}$ The administration of suboptimal care in ICU, may be one of the factor affecting the severity scores and successive predicted mortality of admitted patients. ${ }^{8}$

Previously, a multi centric and multinational cohort study was conducted across 35 countries in more than 300 ICUs, to evaluate the SAPS 3 index in general ICU patients. ${ }^{7,8}$ They concluded that SAPS 3 database efficiently quantify the severity of the critical patients admitted in ICU, and its admission score in ICU was able to predict the clinical status at hospital discharge. ${ }^{8}$ However, the authors mentioned that external validation of this index is required in surgical patients. ${ }^{8}$ Discrimination indicates the extent to which the severity score model distinguishes between the survivors and non-survivors. Calibration of a severity score model describes the extent to which the predicted mortality mirrors the observed mortality. The discrimination and calibration is required to analyse SAPS 3 index. In the present study, the SAPS 3 score (cut-off of 42 score) demonstrated $83.3 \%$ of non- survivors versus $54.2 \%$ of survivors, hence demonstrating a fair discriminatory power. The observed mortality $(17.1 \%)$ and predicted mortality $(15.84 \%)$ was $17.1 \%$ and $15.2 \%$ respectively and was in proximity with standardized mortality ratio (SMR) of 1.07. These values demonstrates a good calibration in the study population. We infer that the probability of death increases noticeably with higher SAPS 3 scores. Our results are in agreement with Silva et $\mathrm{al}_{,}{ }^{[4]}$ who evaluated the applicability of SAPS 3 in Brazilian hospital.

The present study has certain limitations. The sample size was small since it was a preliminary study done in limited time frame in a single (10 bedded) ICU. The study population was further reduced owing to the exclusion of nonsurgical patients. In the present study the higher SAPS 3 score was mainly because of patients admitted after gastrointestinal surgery. These patients are mainly operated for perforation peritonitis and are hypovolemic, have severe bacterial infection and frequently have co-morbid associations, particularly involving the respiratory system. In addition, hypovolemia diminish the perfusion of end organs, particularly kidney leading to deranged renal functions. In the present study, the reason of admission in ICU (acute abdomen: gastrointestinal surgeries) and serum creatinine score were particularly high in northern Indian population and was similar to the Brazilian population conducted by Silva et al. ${ }^{4}$ This in turn has resulted in higher values of SAPS 3 score.

SAPS 3 prognostic model was initially developed from data of general ICU population pool and it may not be the precise representative of specific population. ${ }^{7,8,11}$ In the present study, we have not customized the equation of SAPS 3 prognostic model but follow the standard global equation for postsurgical patients admitted in ICU. Investigators in the 
past, conducted studies to externally validate the customized equation of SAPS 3 prognostic admission model in patients admitted in critical care. ${ }^{9-11}$ They observed that customized SAPS 3 equation was though comparable in terms of discrimination with standard equation but certainly has improved calibration and may provide more precise estimation of SMR closer to unity. ${ }^{9-12}$ Further studies with larger sample size are certainly required for external validation of customised SAPS 3 index in postsurgical Indian patients admitted in ICU, to obtain more beneficial results in particular local settings.

SAPS 3 prediction index is exclusively based on data collected in first hour of ICU admission therefore applicable for ICU triage in postoperative patients, as it displays the clinical status of patient and not the standard of care as seen with other prediction models. ${ }^{12,13}$ In addition, it may also be applied in preoperative period in high risk surgical patients to predict severity of illness and risk of postoperative complications requiring ICU admission. ${ }^{14}$

\section{Conclusion}

In the present study it was observed that SAPS3 prognostic index demonstrated reasonable discriminatory power with ability to distinguish survivors and non survivors. SAPS 3 scoring system seems to be a useful prognostic tool for acute surgical patients admitted in ICU. However, a larger subset of surgical patients with inclusion of multicentric ICUs is certainly required to establish its prognostic applicability in Indian population. The scoring system is easy to calculate, having readily available electronic applications, without need of complex analysis and may be routinely applied in critical care settings, to stratify the risk associated in surgical patients.

\section{Funding source(s) of support: Nil}

\section{Presentation at a meeting: Nil}

Conflicting Interest: Nil. ("I declare that I have no financial or personal relationship(s) which may have inappropriately influenced me in writing this paper").

\section{Acknowledgement: Nil}

\section{References}

1. Knaus WA, Wagner DP, Draper EA, Zimmerman JE, Bergner M, Bastos PG. The APACHE III prognostic system: risk prediction of hospital mortality in critically ill hospitalized adults. Chest 1991;100:1619-36.

2. Le Gall JR, Lemeshow S, Saulnier F. A new simplified acute physiology score (SAPS II) based on a European/North American multicenter study. JAMA1993;270:2957-63

3. Cook R, Cook D, Tilley J, Lee K, Marshall J; Canadian Critical Care Trials Group. Multiple organ dysfunction: baseline and serial component scores. Crit Care Med 2001;29:2046-50.

4. Silva Junior JM, Malbouisson LM, Nuevo HL, Barbosa LG, Marubayashi LY, Teixeira IC et al. Applicability of the simplified acute physiology score (SAPS 3) in Brazilian hospitals. Rev Bras Anestesiol. 2010;60:2031.

5. Bennett-Guerrero E, Hyam JA, Shaefi S, Prytherch DR, Sutton GL, Weaver PC et al. Comparison of PPOSSUM risk- adjusted mortality rates after surgery between patients in the USA and the UK. Br J Surg 2003;90:1593-98.

6. Marshall JC, Cook DJ, Christou NV, Bernard GR, Sprung CL, Sibbald WJ. Multiple organ dysfunction score: a reliable descriptor of a complex clinical outcome. Crit Care Med 1995;23:1638-52.

7. Metnitz PG, Moreno RP, Almeida E, Jordan B, Bauer $\mathrm{P}$, Campos RA et al. SAPS 3 - From evaluation of the patient to evaluation of the intensive care unit. Part 1: Objectives, methods and cohort description. Intensive Care Med 2005;31:1336-44

8. Moreno RP, Metnitz PG, Almeida E, Jordan B, Bauer P, Campos RA et al. SAPS 3 - From evaluation of the patient to evaluation of the intensive care unit. Part 2: Development of a prognostic model for hospital mortality at ICU admission. Intensive Care Med 2005;31:1345-55

9. Soares M, Salluh JI. Validation of the SAPS 3 admission prognostic model in patients with cancer in need of intensive care. Intensive Care Med 2006;32:1839-44.

10. Nassar AP, Malbouisson LM, Moreno R. Evaluation of Simplified Acute Physiology Score 3 performance: a systematic review of external validation studies. Crit Care. 2014;18:R117.

11. Lim SY, Koh SO, Jeon K, Na S, Lim CM, Choi WI, et al. Validation of SAPS3 admission score and its customization for use in Korean intensive care unit patients: a prospective multicentre study. Respirology. 2013;18:989-95.

12. Sakr Y, Krauss C, Amaral AC, Réa-Neto A, Specht M, Reinhart K et al. Comparison of the performance of SAPS II, SAPS 3, APACHE II, and their customized prognostic models in a surgical intensive care unit. $\mathrm{Br} \mathbf{J}$ Anaesth 2008;101:798-803.

13. Haq A, Patil S, Parcells AL, Chamberlain RS. The Simplified Acute Physiology Score III Is Superior to the Simplified Acute Physiology Score II and Acute Physiology and Chronic Health Evaluation II in Predicting Surgical and ICU Mortality in the "Oldest Old". CurrGerontolGeriatr Res. 2014;2014:934852.

14. Silva JM Jr, Rocha HM, Katayama HT, Dias LF, de Paula MB, Andraus LM, et al. SAPS 3 score as a predictive factor for postoperative referral to intensive care unit. Ann Intensive Care. 2016;6:42.

15. Balaji B, Rao AB, Kumar VS. Performance of simplified acute physiology score 3 admission score as a predictor of ICU mortality in a tertiary care hospital of rural Telangana, India. International Journal of Advances in Medicine. 2016;3:716-20.

16. Juneja D, Singh O, Nasa P, Dang R. Comparison of newer scoring systems with the conventional scoring systems in general intensive care population. Minerva Anestesiol. 2012;78:194-200.

17. Boyd O, Grounds M (1994) Can standardized mortality ratio be used to compare quality of intensive care unit performance?.Crit Care Med 22:1706-8. 